\title{
Identification of Core Technology of Digital SPC Exchange and Intelligentialize Prospect of Electronic Communication Equipment Industry
}

\author{
Yingxin Zhang1,a \\ ${ }^{1}$ Tianjin University of Commerce Boustead College, 300384
}

\begin{abstract}
SPC switch belongs to the communication system equipment manufacturing class and belongs to the high-tech industry. Mastering the core technology of digital SPC switch is related to communication security and national information security. HuaWei has already mastered the core technology of digital SPC switch, and on this basis has accumulated the core technology capability to develop intelligently. In this paper, patent data is used to analyze the patent family of digital SPC switches at home and abroad, and based on this analysis and identification of core technologies, and finally, the intelligent development direction of the industry is predicted.
\end{abstract}

\section{Core technology identification of digital SPC exchange}

After data de-noising, the number of published patent applications was 18235 as of February $21^{1}, 2017$. Patent data is obtained from Deventer Innovation Index and indexed by keywords and DC code.

In Web of Science, the retrieval condition of selecting Derwent Database II is: digital program control exchange or stored program control exchang or digital stored program control exchange. Can get 2307 results.

This section will use Citespace to map patent family knowledge. In this paper, the analysis results are generated automatically by time line drawing.

\subsection{Convergence of key technical points of digital SPC exchange}

Based on the DCs code of Deventer code as the keyword, the mixed co-occurrence network is drawn with the emergent words as shown in figure 1. In the figure, the first 11 largest clusters are represented by circles. Digital video tape recorder; Comparative performance - storage devices; The antenna; Service cycle; Microprocessors; Minimum priority; Coupled - remote image monitoring system. The patented technology mainly includes the T01 (Digital Computers) Digital computer; W01 (Telephone and Data Transmission Systems) Telephone and Data Transmission system; W02 (Broadcasting, Radio and

\footnotetext{
1 Corresponding author: zhangyingxin857@126.com. associate professor. Research Field: Disruptive technology. Project support: Tianjin science and technology project (Item NO.:17ZLZXZF00510)
}

Line Transmission Systems) Broadcasting, Radio and linear Transmission Systems; W04 (Audio/Video Recording and Systems) Audio/Video Recording and system; W03 (TV and Broadcast Radio Receiver) TV and Broadcast Radio Receiver; U21 Logic Circuits, Electronic Switching and Coding, Basic Logic Circuits; Computer Peripheral Equipment (T04); U24 (Amplifiers and Low Power Supplies) Amplifiers and Low Power Supplies; T06 (Process and Machine Control) Process and Machine Control; U14 memory, Film and Hybrid Circuits, Digital Memories. U13(Integrated Circuits) Integrated Circuits. The circle size in the figure indicates the frequency of key technology clustering. Therefore, control computing radio communications equipment; Digital video tape recorder; Comparative performance - storage devices; The antenna; Service cycle; Microprocessors; Minimum priority; Coupled - remote image monitoring system is a key technology point in digital SPC switch.

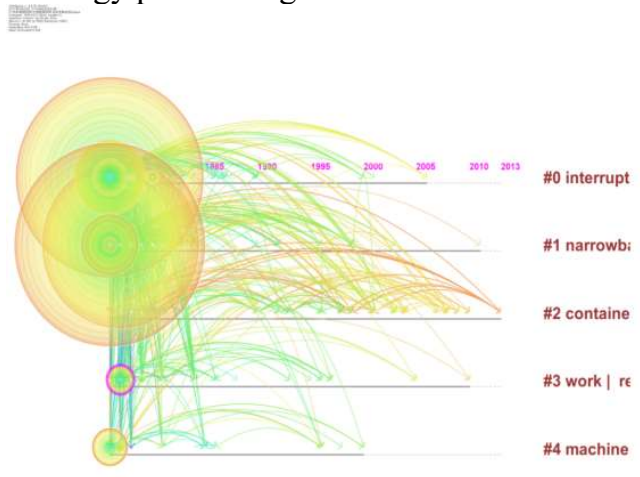

Figure 1. Patent family knowledge atlas of digital SPC exchange. 


\subsection{Measurement of digital SPC exchange technology co-class partners, TCP}

Bibexcel, a large document processing software, is used to process the sample data here. Can get the common matrix. Through calculation of the value from the table, each technology types in many other types exist between class relations. The size of the kind of partner reflects a technology type to other type the link between the scope. Technical class partner of numerical value is greater the technical types and many other link between technology type.

The "technology co-classification index" is introduced here, which can be used to measure the correlation between technology types. The Jaccard coefficient proposed by Leydesdorff is used to standardize the technical common type matrix, and the technical common type index matrix is obtained. Compared with the Saltom cosine coefficient, Jaccard coefficient is more suitable for calculating the co-citation strength or co-occurrence strength matrix. This paper refers to the calculation of luan chunjuan revised common index as follows:

$$
S(i, j)=\frac{\operatorname{coo}(i, j)}{\operatorname{occ}(i)+o c c(j)-\operatorname{coo}(i, j)}
$$

Where, $S(I, j)$ represents the common index of technical classification $I$ and $j, \operatorname{coo}(I, j)$ represents the common frequency of $\mathrm{I}$ and $\mathrm{j}$, and $\operatorname{occ}(\mathrm{I})$ and $\operatorname{occ}(\mathrm{j})$ represent the frequency of occurrence of $I$ and $j$ respectively.

The value range of technology co-class index (TCI) is between 0 and 1 . The larger the value, the more common the commonness between I and $\mathrm{j}$, and the stronger the correlation.

After calculation, table 1 technology common partner matrix table is obtained. In the table, the core technology of Digital SPC exchange is expressed as: 1-t01 (Digital Computers) Digital computer; 2-W01 (Telephone and Data Transmission Systems) phone and Data Transmission system; 3-W02 (Broadcasting, Radio and Line Transmission Systems) Broadcasting, Radio and linear Transmission Systems; 4-W04 (Audio/Video Recording and Systems) Audio/Video Recording and system; 5-W03 (TV and Broadcast Radio Receiver) TV and Broadcast Radio Receiver; 6-U21 (Logic Circuits, Electronic Switching and Coding, Basic Logic Circuits)7-t04 (Computer Peripheral Equipment); 8-u24 (Amplifiers and Low Power Supplies) Amplifiers and Low Power Supplies; 9-t06 (Process and Machine Control) Process and Machine Control; 10-U14 (Memories, Film and Hybrid Circuits, Digital Memories) memory, Film and Hybrid Circuits, Digital Memories; 11-u13 (Integrated Circuits) Integrated Circuits. The Numbers in the column of TCP in the following table respectively represent the number of technical common partners.
Table 1. Technology common partner matrix.

\begin{tabular}{|c|c|c|c|c|c|c|c|c|c|c|c|c|}
\hline & 1 & 2 & 3 & 4 & 5 & 6 & 7 & 8 & 9 & 10 & 11 & TCP \\
\hline 1 & 577 & 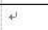 & 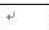 & 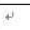 & 4 & 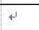 & 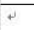 & 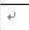 & 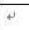 & 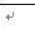 & 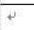 & 0 \\
\hline 2 & 220 & 259 & 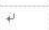 & 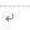 & 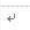 & 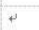 & 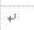 & 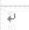 & 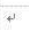 & 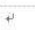 & 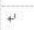 & 1 \\
\hline 3 & 280 & 76 & 61 & + & 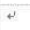 & 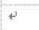 & 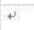 & & + & + & 4 & 2 \\
\hline 4. & 143 & 80 & 100 & 5 & + & 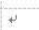 & 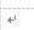 & & + & 4 & 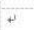 & 3 \\
\hline 5 & 109 & 0 & 0 & 0 & 0 & 4 & 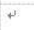 & $\pi$ & 4 & + & 4 & 1 \\
\hline 6 & 164 & $0+$ & 0 & 0 & 0 & 0 & + & 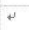 & 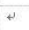 & 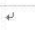 & + & 1 \\
\hline 7. & 91 & 0. & 0 & 0. & & 73 & 0. & & 4 & 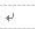 & 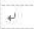 & 2 \\
\hline & & 0 & & & & 0 & & & & & 3 & 20 \\
\hline & & 0. & 0. & & & & & & 5. & & . & 2 \\
\hline & & 0 & & & & & & & & & & 2 \\
\hline 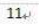 & 46 & 0 & 0 & 0 & 0 & 49 & 4 & 0 & 0 & 9 & 14 & 4. \\
\hline
\end{tabular}

Furthermore, according to the formula (1) to calculate, the correlation between each component technology of digital SPC switch core technology is calculated, and the technology common index matrix is obtained as shown in table 2 .

\subsection{Measurement of digital SPC exchange technology co-classification index, TCI}

The technology co-classfication reveals the degree of correlation between two technology types. The technical common index (TCI) is obtained by calculation. The specific calculation table is as follows.

Table 2. Technical common exponential matrix.

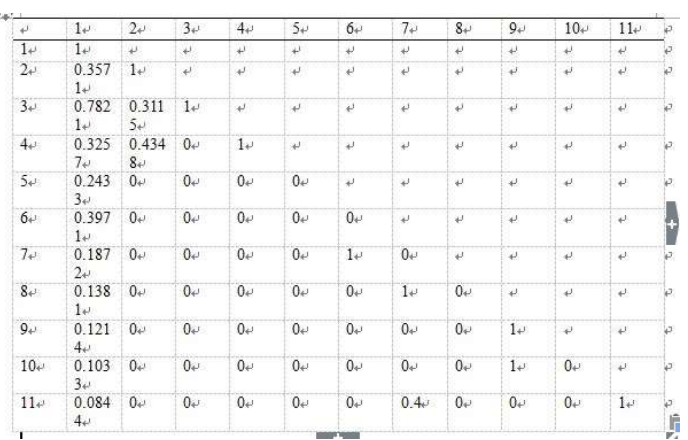

Obviously, the technology common index of W01 telephone and data transmission system and T01 digital computer is 0.3571 . The technology common index of W02 broadcast, radio and linear transmission system and T01 digital computer is 0.7821 . The technical common index of W02 broadcast, radio and linear transmission system and W01 telephone and data transmission system is 0.3115 . The technical common index between W04 audio/video recording and system and T01 digital computer is 0.3257 ; The technical common index between W04 audio/video recording and system and W01 telephone and data transmission system is 0.4348 ; The technology common index between U21 logic circuit, electronic switch and code and T01 digital computer is 0.3971 .

In conclusion, from a static perspective, although the size of the common index varies among different technologies, the technical common index is expressed as the close degree of technical correlation, which means that the development of core technologies should focus on the correlative development of these technologies.

Then, how to cluster among these key technologies can be obtained by clustering analysis below. 


\subsection{Digital SPC exchange core technology subcluster and features}

The technical co-classification matrix generated by Bibexcel was imported into ucinet, and the condensed subgroup was carried out by CONCOR method.

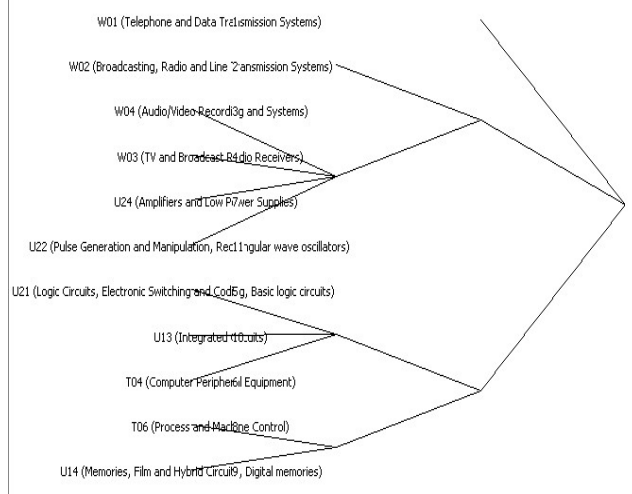

Figure 2. Condensed subgroup of digital SPC exchange.

Through the analysis of condensed subgroups (clustering), three directly related subgroups are formed, respectively:

(1) W04; W03.U24; U22 means (Audio/Video Recording and Systems) Audio/Video Recording and system; TV and Broadcast Radio Receiver Amplifiers and Low Power Supplies are high-level technology groups.

(2) U21; U13; T04 is Logic Circuits, Electronic Switching and Coding, Basic Logic Circuits. Integrated circuits and computer peripherals are high-level technology groups.

(3) T06; U14 is process and machine control; Memory, film and hybrid circuits and digital memory are highly relevant groups of technologies.

These direct contact nodes constitute a strong relationship and keep the relationship of subgroup within the function of strong relationship is, and also through some bridge between subgroups W01 telephony and data conversion system, and W01 broadcasting, radio and linear transformation system to form weak relationship, weak relationship role makes greater coherence in the structure of network. In short, the existence of weak relationships determines the existence of the entire network.

If Chinese enterprises want to master the core technology in this field independently, they should not only overcome the technology of building strong relationship among sub-groups, but also grasp the bridge technology in the bottleneck position and weak relationship.

\section{Intelligent development prospect of electronic communication equipment industry}

\subsection{Promote the development of reverse technology with the help of big data}

Industry 4.0 era, with new tools - big data. At the same time, there is a huge industrial market and therefore a huge database. The design that needs to be done and the technology that needs to be developed can be inferred back through big data, that is, reverse technology development.

The specific approach is as follows: firstly, from a horizontal perspective, it is to collect the technical data of related enterprises involved in the technical correlation analyzed above, and integrate them into the database of the core technologies of the electronic communication equipment industry. From a vertical perspective, the integrated core technology research and development data covers the whole process from design, material selection, manufacturing, testing, operation and maintenance to the end of the life cycle. Second, there are high development requirements for accuracy and reliability in the electronic communications equipment industry, so data on failures or operational anomalies should be collected even if the probability is low. By using these big data and big data analysis technologies to catch data generated during operation anomalies, defects and problems can be found through analysis, so as to improve accuracy and reliability.

Make full use of imported foreign advanced communication equipment, and let data guide innovative personnel to design and manufacture components through reverse technology. Due to the existence of a large amount of dark mute knowledge, big data can provide a new method of mining these recessive technologies.

\subsection{The equipment pre-diagnosis and health management techniques were used to analyze the engineering data flow}

The implementation process of this technology is a data-driven intelligent analysis system. It mainly includes five steps: data collection, feature extraction, performance prediction and performance visualization, and performance diagnosis. See figure 3 data engineering process analysis diagram.

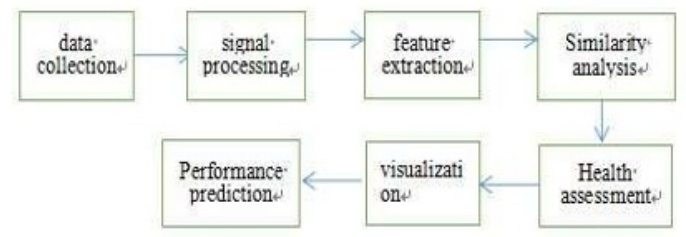

Figure 3. Data engineering process analysis diagram

Through the use of sensor signals, status monitoring data, maintenance of historical records and so on. The above data are processed by feature extraction, and the recession characteristics are obtained. Based on performance characteristics, production system performance can be evaluated and quantified by health confidence values. The university of Cincinnati NSF intelligent maintenance "industry-university-institute" cooperation research center IMS proposed and developed for tool kit, in the process of the diagnosis and health management will be widely used in the intelligent 
analysis algorithm together, each algorithm under different circumstances and evaluate the advantages and disadvantages, adopt a systematic approach to priority of the applicable degree of each algorithm, thus reducing the number of trial and error in the actual application development

\section{Conclusion}

From the perspective of patent analysis and with the help of patent co-classificaiton analysis method, this paper takes digital SPC switch as the research object and identifies the composition of its core technology and the strong and weak relationship between its sub-technologies. Combined with the background of China's vigorous development of industry 4.0, the prospect of intelligent development of communication equipment industry is prospected.

\section{References}

1. Björn A. Sandén and Christian Azar, 2003, Near-term technology policies for long-term climate targets, Submitted for publication in Energy Policy, Oct, 2003,
Chalmers University of Technology, Göteborg, Sweden.

2. Christophe E C, Jessie P, Michel K. The influence of individual, contextual, and social factors on perceived behavioral control of information technology: A field theory approach [J]. Journal of Management Information Systems, 2011, 28(3): 201-234.

3. Giovanni Dosi. Technological paradigms and technological trajectories [J]. Research Policy 11(1982) B, 147-162.

4. Patel\&Pavitt. The continuing, widespread (and neglected) importance of improvements in mechanical technologies [J]. Research Policy, Volume 23, Issue 5, September 1994, Pages 533-545.

5. Blake E. Ashforth and Fred Mael. Social Identity Theory and the Organizaion [J]. Social Identity Theory and the Oragnizaion [J]. The Academy of Management Review, Vol.14, No.1, pp.20-39.

6. Jungho Kim, Chang-Yang Lee, Yunok Cho. Technological diversification, core-technology competence, and firm growth [J]. Research Policy, 2016(45):113-124 